\title{
Mapping Meta-Therapy in Voice Interventions onto the Rehabilitation Treatment Specification System
}

\author{
Leah B. Helou, Ph.D., CCC-SLP, ${ }^{1}$ \\ Jackie L. Gartner-Schmidt, Ph.D., CCC-SLP, ${ }^{2}$ \\ Edie R. Hapner, Ph.D., CCC-SLP, ${ }^{3}$ \\ Sarah L. Schneider, M.S., CCC-SLP, ${ }^{4}$ and \\ Jarrad H. Van Stan, Ph.D., CCC-SLP' $5,6,7$
}

ABSTRACT

${ }^{1}$ Department of Communication Science \& Disorders, University of Pittsburgh, Pittsburgh, Pennsylvania; ${ }^{2}$ University of Pittsburgh Voice Center, Department of Otolaryngology, University of Pittsburgh Medical Center, Pittsburgh, Pennsylvania; ${ }^{3}$ Department of Otolaryngology, UAB Voice Center, University of Alabama, Birmingham, Birmingham, Alabama; ${ }^{4}$ UCSF Voice and Swallowing Center, Department of Otolaryngology Head and Neck Surgery, University of California, San Francisco; ${ }^{5}$ Department of Surgery, Harvard Medical School, Boston, Massachusetts; ${ }^{6}$ Massachusetts General Hospital, Center for Laryngeal Surgery and Voice Rehabilitation, Boston, Massachusetts; ${ }^{7}$ Department of Communication Sciences and
Disorders, MGH Institute of Health Professions, Boston, Massachusetts.

Address for correspondence: Leah B. Helou, Ph.D., CCC-SLP, 6075 Forbes Tower, 3600 Forbes Avenue, Pittsburgh, PA (e-mail: 1bh7@pitt.edu).

Clinical Expertise for the Delivery of Voice Therapy InPerson and through Telepractice; Guest Editor, Maria Dietrich, Ph.D., CCC-SLP

Semin Speech Lang 2021;42:5-18. (C) 2021. Thieme. All rights reserved. Thieme Medical Publishers, Inc., 333 Seventh Avenue, 18th Floor, New York, NY 10001, USA DOI: https://doi.org/10.1055/s-0040-1722756.

ISSN 0734-0478. 
veying information with the goal of appropriately shaping the patient's mental representations, and are delivered with verbal cues, stories, analogies, etc. This manuscript provides specific examples of how metatherapy is applied in clinical voice practice. Considerations for future investigation of meta-therapy are proposed.

KEYWORDS: meta-therapy, Rehabilitation Treatment Specification System, voice therapy, treatment theory, expectations

Learning Outcomes: As a result of this activity, the reader will be able to (1) describe the treatment theory and self-referential features of meta-therapy; (2) explain how meta-therapy relates to the Rehabilitation Treatment Specification System; (3) describe three targets of meta-therapy.

Research in voice therapy has compared some of the following categories: service delivery models, ${ }^{1-4}$ duration of voice therapy sessions, ${ }^{5,6}$ number of voice therapy sessions, ${ }^{6-10}$ and type of voice therapy programs. ${ }^{11-14}$ Across these categories of inquiry, similar outcomes are shown regardless of the variations in therapy. Perhaps one element behind this consistency is meta-therapy. Meta-therapy in voice treatments is believed to be one core determinant of successfully changing vocal behavior that transcends therapy type, duration of session, number of sessions, and delivery model. Specifically, meta-therapy is the conversation during voice therapy that shapes the therapeutic process and patient response to that process. ${ }^{15,16}$ The qualitative and quantitative aspects of meta-therapy are likely most different when comparing treatment delivered by experienced versus novice clinicians, and practically more similar when comparing treatment across multiple experienced clinicians. Essentially, it is thought that master clinicians have developed well-used dialogues that focus and guide their patients' perspectives, building a conceptual framework for what we are doing here in voice therapy. ${ }^{15-20}$ These dialogues have also been referred to as the Language of voice therapy, the How of voice therapy, and an important component part of the It factor. ${ }^{21-24}$ Collectively, such dialogues have the power to overcome the inaccurate expectations that patients often bring into the therapy setting, and which often serve as barriers to success.
Unfortunately, the current colloquial narrative of meta-therapy is probably not specific enough to allow rigorous research studies, replicate or adopt in clinical care, or impart to other clinicians and students during training/education. ${ }^{21-23}$ Oftentimes, traditional treatment narratives contain all of the intervention's essential information, but lack critical elements that make the narrative interpretable and/or useable by others. $^{24,25}$ These missing critical elements include: (1) standardly defined and operationalized terminology, (2) explicit identification of the clinical actions that are believed to be responsible for the treatment's effects, and (3) connecting the individual (or groups) of clinician action(s) to their respective clinically meaningful changes in patient functioning. ${ }^{26,27}$ These three elements are essential because they enable clinicians to (1) clearly understand the treatment description, (2) know what actions they must enact to provide the treatment, and (3) test whether or not their actions were clinically successful, respectively.

The aim of this paper is to explicitly identify, and then standardly operationalize, the clinician actions, modified patient functions, and key constructs ostensibly responsible for the effects of meta-therapy. Meta-therapy will be characterized through the lens of a standardized, cross-disciplinary framework for identifying the critical aspects of all rehabilitation interventions called the Rehabilitation Treatment Specification System (RTSS). ${ }^{28,29}$ (The Manual for Rehabilitation Treatment Specification is available at https://acrm.org/ 
acrm-communities/rehabilitation-treatmentspecification/manual-for-rehabilitation-treatment-specification/ and describes the RTSS specification process in detail. All terms that have definitions in the open-access Manual will be underlined when first used.).

\section{META-THERAPY AS TREATMENT THEORY}

As the prefix "meta-" denotes (e.g., "situated behind or beyond" and applied to such fields as meta-physics, meta-psychology, and meta-philosophy), meta-therapy pertains to aspects of voice treatments that might be "beyond" the immediately observable. ${ }^{30}$ In other words, some meta-aspects of voice treatment are purely rational (i.e., ideas or theories), existing only in the clinician's mind. ${ }^{31}$ Previous offerings on meta-therapy stated that voice treatment should address the patient's "conceptual framework about what we are doing here in voice therapy," and highlighted the fact that the framework a clinician helps a patient to build is a direct extension of the conceptual framework built for that clinician in the course of their own training and experience. ${ }^{15-20}$ Essentially, conceptual frameworks are based upon basic ontological assumptions. ${ }^{32}$ Ontologies represent some subject area or domain-voice interventions in this case- as a set of concepts and the relationships among those concepts. Because ontologies identify the relevant concepts of a context or domain, they direct individuals (i.e., the patient and clinician) toward what should be attended to and what should be ignored during the course of voice therapy. Therefore, ontologies essentially shape how patients view their voice and how their voice interacts with the world around them. Additionally, ontologies shape how clinicians view the treatment they deliver and how they should interact with the patient.

The "conceptual framework" of meta-therapy (that is hoped to be imparted to the patient) has a parallel in the RTSS, as the RTSS is based on the clinician's treatment theory. A treatment theory is defined as a "conceptual system that predicts the effects of the ingredients used on their targets, specifying the law(s) of the relationship between ingredients and changes in targets." As shown in Fig. 1, all RTSS-based treatment theories are made up of a 3-part treatment component: ingredient(s) (clinician action(s) that directly changes a patient function), target (a singular patient function that is directly changed by the ingredient(s) delivered), and the mechanism(s) of action (how the ingredient(s) is expected to directly affect the target). These three concepts within a treatment component have a causal relationship because, once the clinician delivers an ingredient, it begins a cascade

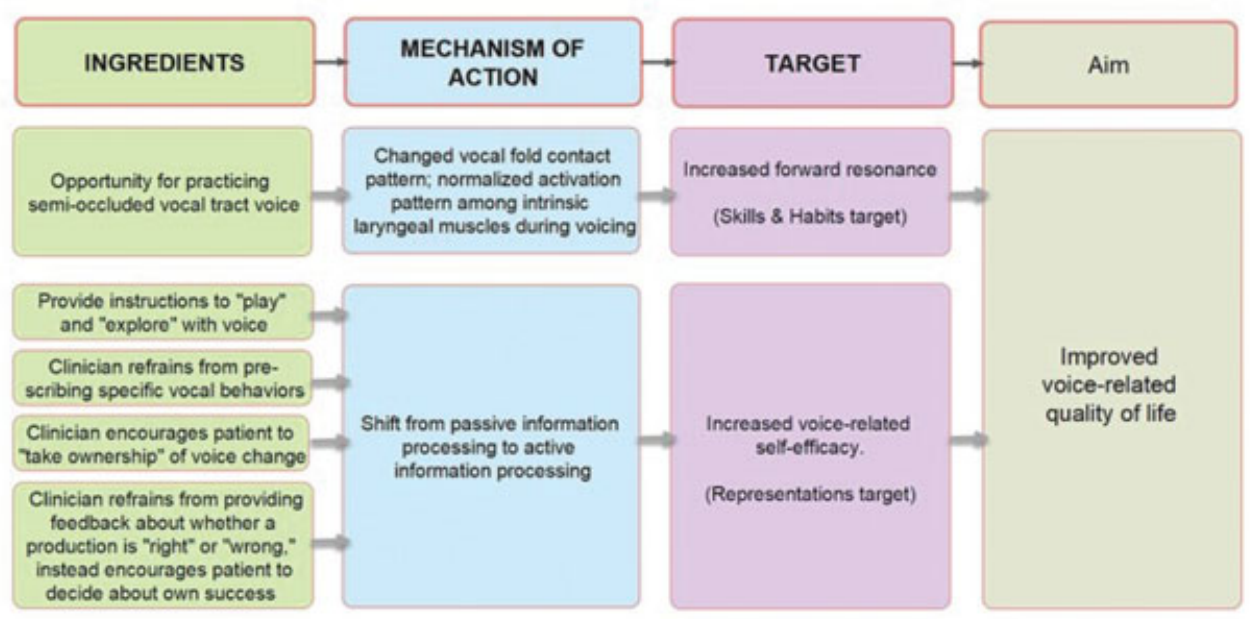

Figure 1 Three-part treatment component structure of the Rehabilitation Treatment Specification System. Examples of clinical Ingredients, Mechanisms of Action, and Targets are given in service to one specific clinical Aim. 
of changes in patient functioning (i.e., mechanisms of action) that end in the desired modification of a single patient function (i.e., the target).

If the aim of measuring something in a research study is to better understand how an ingredient affects a target, this approach is aligned with the RTSS concept of "mechanisms of action" (reasons why we think an ingredient will affect a target). For example, a clinician may provide opportunities for the patient to practice voicing using a semi-occluded vocal tract (ingredient) in hopes that it will increase forward resonance (target). Many other patient functions may be modified by this ingredient and (in this example) are in the mechanisms of action; e.g., vocal fold contact patterns during phonation, ${ }^{33}$ impedance matching between the source and the filter, ${ }^{34}$ or normalized thyroarytenoid-cricothyroid muscle activation. ${ }^{34}$ While changes in vocal fold contact, impedance matching, and intrinsic laryngeal muscle activation are hypothesized to occur, they are not (in this example) measured during routine treatment. Rather, they are only assumed to have changed in a specific way because of changes (e.g., vocal, resonant, physical) that are detectable to the clinician and/or patient. Additionally, increases in forward resonance must occur for the practice to be considered successful. If changes to vocal fold contact, impedance, or muscle activation occur without increased forward resonance (target), the ingredient (practice) is considered to have not worked. As illustrated in Fig. 1, the RTSS defines treatment aims as changes in patient functioning that are indirectly affected by ingredients' effect of targets. For example, increased resonance in voicing (target) may help improve the patients voice-related quality of life (aim). Thus, the effects of ingredients on aims are indirect and achieved only through their influence on intermediate targets.

As the terms "treatment theory" or "ontology" or "conceptual framework" denote, this meta-aspect of voice treatment directs the clinician and patient toward what should be observed during treatment and how those observations should be described/measured. While some voice treatment ingredients may be entirely measurable without theoretical context (e.g., the act of providing the patient with a personal amplification device ${ }^{35}$ ), many ingredients need a theoretical context to know how they should be measured (e.g., repetitive voicing could be a resistance exercise to increase strength/endurance of a voice-related muscle or opportunities to practice a vocal skill). Targets of voice treatment, like all behavioral interventions, also often need a theory to be identified with clarity.

In states of non-specific theory, the patient will remain unclear about (or will be forced to come up with) their own conceptual model about what constitutes successfully modified voicing, what they should be doing during/outside the treatment sessions, and what their role is in the treatment process. Suppose a clinician provided a patient an amplification device; what is the purpose of providing the device? The target of the amplification device may be obvious as "increased sound intensity" when the context is a patient with bilateral vocal fold paralysis and pathologically reduced vocal intensity. However, the target of the amplification device may be less obvious when the context is a teacher with bilateral vocal fold nodules. Is the desired target passive increases in the teacher's loudness, or reduced excessive loudness, or decreased vocal fatigue throughout their workday, or something else? The treatment theories contained in metatherapy may be a potential antidote to the risk of patients being left to conjure their own conceptual models. In essence, many times (but not all the time) an observer must know the clinician's treatment theory to observe treatment and correctly identify the aspects of therapy that are provided.

Treatment theories must be testable through observation/measurement, as they predetermine how a treatment can be evaluated for efficacy (i.e., performance of an intervention under ideal and controlled circumstances) or effectiveness (i.e., performance under'real-world' conditions). Specifically, treatment theories identify which aspects of the treatment session are to be considered ingredients and targets, and the relationship between the two (the mechanism of action). When lacking a treatment theory, the clinician's "treatment" would be non-specific and risk being "cookbook" (e.g., "lip trills will generally help your voice") and not testable (e.g., questions like "why will lip trills help?" and "what specifically will lip trills change 
in the voice" are not answerable). Without a treatment theory, the connection between changes (or lack of changes) in voice measures after voice therapy become circular. For example, a clinician could simply measure everything possible about the voice (e.g., fundamental frequency, sound pressure level, jitter, shimmer, cepstral peak prominence, etc.) without establishing a connection to the treatment. Then the clinician could use whichever measures changed as "proof" that the therapy "worked," regardless of the relationship between the measured changes and the ingredients provided. In sum, the use of the prefix "meta" stems in part from the fact that meta-therapy describes theoretical elements of (or "beyond") vocal rehabilitation, which require empirical observation for support or falsification.

\section{META-THERAPY AS SELF- REFERENTIAL}

The prefix "meta" is also self-referential. Applying this sense of the term, meta-therapy can be expressed as "[therapy] about voice \{therapy\}" or "[treatment] about voice \{treatment\}." Here, the [square] and \{curly\} brackets denote [meta] and \{non-meta\} treatment aspects. Previous writings on meta-therapy state that it involves a clinical dialogue that supports the patient's understanding of the therapeutic approach. ${ }^{15-17}$ That clinical dialogue serves as a substrate that helps the patient build a conceptual framework about "what we are doing here in therapy." There appear to be five self-referential goals of metatherapy, which involve modified cognitions, knowledge, beliefs, attitudes, intentions, and/or awareness about (1) the process of vocal improvement during treatment (e.g., failure is part of the process, progress is not linear); (2) the patient's role in treatment (e.g., patients are active participants); (3) the role of self-efficacy in treatment (e.g., patients believe that they can control their respiration, voice, etc.); (4) how the patient's relationship between their voice and sense of identity can affect treatment (e.g., the "new" therapeutically established voice might not feel readily compatible with their view of themselves); and (5) how affective states can influence treatment (e.g., recognize when voice changes are related to stressful situations and become able to interrupt unhelpful patterns).

As previously stated, the RTSS defines the smallest meaningful unit of the term "treatment" as a three-part treatment component (e.g., ingredients, mechanism(s) of action, and target) based on treatment theory. The RTSS states that there are generally three types of treatment components reflecting three main types of mechanisms of action: (1) Organ Functions, (2) Skills and Habits, and (3) Representations. Organ Functions treatment components change the efficiency of (or replace) organs or organ systems; e.g., applying pressure on muscle to decrease activation; exercises to increase endurance; place a tracheoesophageal prosthesis to replace the voice source. Skills and Habits treatment components improve mental (e.g., memory, language) or behavioral abilities and support the formation of habits; e.g., providing opportunities to practice voicing to decrease breathy voice quality. Representations treatment components change mental representations such as thoughts, feelings, and volitional behavior; e.g., written instructions to increase knowledge or motivational information to increase the likelihood that the patient will perform an exercise as prescribed. (Fig. 2)

Meta-therapy as a self-referential concept does not appear to map onto the Organ Functions (e.g., resistance training to effect resistance training) or Skills and Habits (e.g., practice to effect practice) treatment components. Also, the five self-referential goals of meta-therapy (see Figure 2) all pertain to changes in the patient's mental representations. Therefore, meta-therapy as a self-reference most clearly aligns with Representations treatment components. These treatment components include the provision of information (ingredients) in hopes of modifying the patient's mental representations about treatment (targets); e.g., cognitions, knowledge, beliefs, attitudes, intentions, and/or awareness to perform volitional behaviors. These shifts in mental representations occur along a continuum of requiring no overt action (e.g., knowledge for knowledge's sake) to requiring overt action (e.g., increased motivation to practice therapeutic voicing in daily life). The RTSS labels the "action" side of this continuum as volition; where 


\section{Goals of Meta-therapy:}

Modified cognitions, knowledge, beliefs, attitudes,

intentions, and/or awareness about:

(1) the process of vocal improvement during treatment;

(2) the patient's role in treatment;

(3) the role of self-efficacy in treatment;

(4) how the patient's relationship between their voice and identity can affect treatment; and

(5) how affective states can influence treatment

Figure 2 Summary list of five goals of meta-therapy.

volition is roughly equated with effort expended by the patient.

Now, if we replace the self-referential definition of meta- therapy ("[treatment] about \{treatment\}") with the RTSS terms, this phrase becomes "[Representations treatment component] about [insert Organ Functions, Skills and Habits, or Representations treatment component\}." To further break this down, the phrase would become "[providing informational ingredient(s) to change the patient's cognitions, knowledge, beliefs, attitudes, intentions, and/or awareness] about [insert Organ Functions, Skills and Habits, or Representations treatment component\}." In fact, applying the RTSS framework, the five selfreferential goals of meta-therapy can be tied to three main types of targets: (1) increasing the accuracy (and/or amount) of knowledge about what is going to happen in voice therapy, (2) changing patient beliefs or attitudes about their voice that may "facilitate" or "get in the way" of therapeutic progress, and (3) increasing the probability that the patient will perform therapeutic activities as directed outside of the therapy session (i.e., "volition"). Notably, the third target type can be related to the concept and work of voice therapy adherence. Previous work on adherence to voice therapy echoes some of the themes described herein, ${ }^{36}$ in a manner somewhat suggestive of patients' attunement to the value of meta-therapy.

Here, some examples of meta-therapy should be useful to consider. Table 1 illustrates examples of meta-therapy generated by $\mathrm{co}^{-}$ authors Helou, Gartner-Schmidt, Hapner, and Schneider. These examples are highly condensed versions of clinical narratives, analogies, "spiels," and other variants on clinical dialogue that are commonly employed by these clinicians with voice therapy patients. While informational ingredients might be simply provided in a purely didactic manner (e.g., explaining to a patient in physiological terms why excessive laryngeal muscle tension prevents optimal voice use), there is evidence that couching this information in an analogy or metaphor could improve information retrieval $^{37}$ (e.g., discussing how voicing with excessive laryngeal muscle tension "is like driving a car with the brakes on"; credit to van Mersbergen $^{38}$ for this analogy). The examples in Table 1 will also be useful references for the following section, which discusses meta-therapy's focus on issues beyond vocal outcomes per se.

\section{META-THERAPY AS BEYOND MODIFIED VOICING}

Previous offerings about meta-therapy in voice treatments discuss how it encompasses the patient's psychological relationship with their voice, ${ }^{15-20}$ noting that a shift in this relationship can facilitate and underlie observed vocal improvements. Multiple aspects of how the patient views and interacts with their voice have been noted by master clinicians as likely mediating therapeutically desirable changes in voicing, even interfering with showing up for appointments and/or completing the entire prescribed course of voice treatment. ${ }^{12,36,39,40}$ 
Table 1 Goals and Examples of Meta-therapy. Boxes Marked X Indicate Goals of Meta-therapy that Might be Addressed for Each Item

\begin{tabular}{|c|c|c|c|c|c|}
\hline \multirow{2}{*}{\multicolumn{2}{|c|}{ Example statement or dialogue-based theme }} & \multicolumn{4}{|c|}{ Meta-therapy goals } \\
\hline & & \multirow{2}{*}{1} & \multirow{2}{*}{2} & \multirow[t]{2}{*}{3} & \multirow{2}{*}{$\begin{array}{ll}4 & 5 \\
\times & \end{array}$} \\
\hline 1. & $\begin{array}{l}\text { "We are learning some exercises that you will practice, but really what we are } \\
\text { doing is building new patterns that you can use in everyday life; creating vocal } \\
\text { choices; helping you find new ways of connecting with your voice." }\end{array}$ & & & & \\
\hline 2. & $\begin{array}{l}\text { "Voice therapy is more about you developing awareness about the sound and } \\
\text { feel of your voice than me teaching you any exercise or technique." }\end{array}$ & $x$ & $x$ & $x$ & \\
\hline 3. & $\begin{array}{l}\text { "Voice therapy is about guiding you to explore where you feel the sounds. I } \\
\text { am going to consistently ask you two questions after we do different vocal } \\
\text { tasks: Do you feel a difference? Do you hear a difference? You will have to } \\
\text { figure the answers out for yourself, with some gentle guidance by me." }\end{array}$ & $x$ & $x$ & $x$ & \\
\hline 4. & $\begin{array}{l}\text { "Just like those Paint by Numbers activities, your goal is to learn how to Voice } \\
\text { by Feel." }\end{array}$ & & 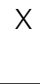 & & \\
\hline 5. & $\begin{array}{l}\text { "In voice therapy, we are working to teach your brain new sensations and } \\
\text { new patterns. At first, it will require conscious awareness. That can be } \\
\text { frustrating but just like learning to type or ride a bike, as the muscle memory } \\
\text { for these new sensations and patterns becomes more habitual, you will not } \\
\text { need to be so conscious of what you do." }\end{array}$ & $\mathrm{X}$ & & & \\
\hline
\end{tabular}

6. Naming the old/default voice and then naming the new/therapy/target voice $\quad X \quad X$ can be a useful practice. For example, "Way to go, you just found your 'Smooth Voice' on your own! Wrap it up, put a bow on it, put it in your pocket, take it home with you, and find it again when you need it. If you can do it once, which you just did, then you can do it again."

7. Probe the patient's narrative around decision-making. If they say "I had to X X yell," clinician might respond with "did you have to yell or did you choose to yell?"

8. Language reveals ownership. If a speaker says, "my voice gets rough...," $X \quad X \quad X$ clinician might encourage them to change their language by adding a second part to their complaint, e.g., "my voice gets rough when I don't use my airflow, when it's stuck in my throat, etc."

9. Acknowledging the natural variability in vocal quality during physical fatigue, X emotional turmoil, cognitive demand, and other affective states can be useful in promoting patience and grace (that the patient affords themself.)

10. "I am the facilitator, but you have to be the implementer. My goal is to make

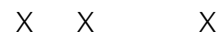
you your own voice therapist. I won't always be saying whether a production was good or not good, or right or wrong. The goal is for you to learn to sense the response that meets your goal (e.g., less effort or a buzzy feel), then get better and better at producing the response you need in the moment."

Note: The five goals of meta-therapy are modified cognitions, knowledge, beliefs, attitudes, intentions, and/or awareness about: (1) the process of vocal improvement during treatment; (2) the patient's role in treatment; (3) the role of self-efficacy in treatment; (4) how the patient's relationship between their voice and identity can affect treatment; and (5) how affective states can influence treatment. 
Further, it is known that psychological aspects such as stress, affect, and self-efficacy can play out in vocal subsystems and in voice itself. . $^{1-46}$ These relationships between the patient's psyche and their voice might extend into impacting the patient's sense of agency (a term which encompasses self-efficacy, locus of control, and other related constructs) toward their voice and voice difficulties. For instance, Gillespie and Verdolini Abbott showed that patients' sense of self-efficacy is related to whether the clinician discusses the origin of their voice disorder in terms of vocal abuse/misuse versus phonotrauma/muscle tension. ${ }^{47}$ In short, it seems apparent that variations in the clinical dialogue (e.g., word choice, terminology, analogies) have the potential to impact outcomes, above and beyond the specific contributions of classical direct and indirect therapy techniques (e.g., opportunities to practice flow phonation and providing vocal hygiene information, respectively).

The patient's psychological relationship with their voice can be addressed in various ways during voice therapy. According to the RTSS, the clinician's treatment theory will dictate how to describe the critical clinician actions (i.e., ingredients), as well as the effects of those actions, on patient functioning (i.e., mechanisms of action, target, or an aim). To concretely illustrate how these aspects of meta-therapy are operationalized depending upon the clinician's treatment theory, consider the ingredient negative practice. ${ }^{39}$ Once the patient repetitively alternates between their baseline voice and their improved voice with as much accuracy as possible, a chain of modified patient functions begin. First, neural activation occurs in the central sensorimotor system; then the peripheral nervous system activates muscles in the head, neck, and respiratory system; then voicing and speech are produced; then feedback contributes to the modification of mental representations like self-efficacy. Among this complex chain of patient functions, some modified function must observably and measurably change so that the clinician action ("negative practice" ingredient) could be considered successful (i.e., effective). This modified function would be the "target." For example, the clinician could measure the "success" of the ingredient opportunities to practice switching between baseline voicing and improved voicing as the target accurate and quick switching between normal and disordered overall voice quality (a skill or habit). The practice ingredient's dosage would be measured according to the number of practice repetitions and progression measured according to the level of difficulty (e.g., automatic speech, reading, spontaneous speech). Another ingredient, provide feedback on performance, would probably also be present with dosing measured by the number of times the feedback was provided. In this case, the target and ingredients identified are not considered "beyond modified voicing" aspects of metatherapy.

In the previous paragraph, the treatment theory regarding negative practice was centered on the premise that alternating between "baseline dysphonia" and "minimal-to-no dysphonia" would improve the patient's vocal skill set and awareness. However, it is possible for a clinician to have a different treatment theory regarding negative practice. For example, perhaps the patient is one who frequently notes that they "can't change their voice," complains that "I never know what's going to come out of my mouth when I open it to say something," and generally feels a low degree of control over their voice. Then, the activity of negative practice could be employed by the clinician as an information ingredient; i.e., provide information about vocal control from the patient themselves. Instead of measuring the accuracy of switching between various vocal productions (i.e., the target of a practice ingredient), the clinician would measure any changes in the patient's voice-related selfefficacy (i.e., the target of an information ingredient). While changes in voicing constitute a modified patient function, this would be in the mechanisms of action for the self-efficacy target, since changes in voicing will not necessarily produce improvements in voice-related selfefficacy. But modified voicing is generally necessary for changes in voice-related selfefficacy to occur. And finally, the success of negative practice (in this example) hinges on whether or not the patient increased his/her voice-related self-efficacy. In this example, negative practice is an aspect of meta-therapy: changing the patient's beliefs or attitudes about their ability to control their own voice (i.e., self-efficacy). 


\section{DISCUSSION}

We used the RTSS to further articulate and operationalize meta-therapy in vocal rehabilitation. Specifically, meta-therapy's conceptual framework of voice therapy was shown to generally align with the RTSS's treatment theory and associated concepts; e.g., the treatment component and its underlying ingredients, mechanisms of action, and target. These metatherapy treatment theories primarily mapped onto the RTSS's treatment category of Representations treatment components. And, the treatment targets in meta-therapy were often focused on changes in the patient's knowledge, affect, and beliefs regarding the therapeutic process. Finally, the ingredients in meta-therapy mainly consisted of clinician actions that conveyed information about the patient's mental representations and were delivered with verbal cues, stories, analogies, etc.

Table 1 presented a limited compilation of meta-therapy goals and dialogue-based examples, all sourced from the first four co-authors' clinical experience. The reader might have noted that this material was not laid within the RTSS framework. More progress must be made in the theoretical articulation of meta-therapy before its finer points can be clearly mapped onto the RTSS. That is, a major limitation of metatherapy in its current form is that it cannot readily be operationalized or standardized. For the meta-therapy goals and associated examples presented in Table 1 to adhere to the RTSS framework, they would need to be rephrased in terms of which patient functions are hypothesized to change directly (i.e., target and mechanisms of action) or indirectly (i.e., aim) from a single (or multiple) clinician action(s). One way of trying to identify a target within a goal is to ask questions such as "What change in the patient would tell me if the treatment ingredient was successful?" or "What am I hoping to clinically achieve by providing this treatment ingredient?" Once the clinician identifies the target (e.g., increased knowledge of the relationship between voice and identity), all patient functions proximal (i.e., closer to basic physiology, like central nervous system activation) to the target fall within the mechanisms of action. Further, all patient functions distal (i.e., closer to activities and participation in daily life, like modified locus of control or improved voice-related quality of life) to the target are viewed as aims.

Consider the ingredient wherein the clinician says "voice therapy is all about guiding you to explore where you feel the sounds." Does the clinician expect the patient to learn this fact and simply recall it in their own words? If so, then the target could be "increased accuracy of knowledge regarding the voice therapy approach." However, perhaps the clinician never asks the patient to repeat this back, and instead is trying to plant a seed that might foster growth in that patient's voice therapy experience. In that scenario, what purpose might this phrase provide? Perhaps it is used as a cue to increase the patient's attention and effort (i.e., volition) to the vocal practice, or their general interpretation of events in the therapy setting. Then, the clinician's statement would be considered a volition ingredient added to the other ingredients of provide opportunities to practice voicing and provide feedback on vocal performance for the target of improved voicing.

The RTSS requires that ingredients, targets, and aims are measurable (i.e., capable of being observed) in routine clinical care. In this context, many of the goals of meta-therapy as currently written may be too generally stated to know how to measure them. Of course, some exceptions exist, such as self-efficacy, which is expected to shift as a function of meta-therapy and is highly measurable. But many of the goals in Table 1 imply a changed patient function from "inappropriate" to "appropriate," but what is specifically meant by "appropriate" was not mentioned. Without more specifically operationalizing "appropriateness," one clinician relying on another clinician's notes for a patient's next session would not explicitly know how to continue the patient's plan of care.

While the meta-therapy examples listed in Table 1 could adhere to an exact phrasing that serves as a measurable ingredient (e.g., how many times is this phrase provided), it is currently unclear which and how many other phrases are paraphrasing the same informational ingredient (i.e., you are an active participant of this therapy) and thus should be categorized together. To be more specific, are the following phrases simply variations of the same informational ingredient?: "helping you find new ways to connect to your voice" and "voice therapy is 
all about guiding you to explore where you feel the sounds" and "I'm basically a giant mirror... you will have to figure this out with some gentle guidance from me." And, how does the clinician know when to increase or decrease the dose (number of times) or intensity (number of times per time period) of these ingredients? These outstanding questions will need to be resolved to more thoroughly characterize meta-therapy in RTSS terms.

\section{FUTURE DIRECTIONS}

In the process of articulating and advancing the concept of meta-therapy in this manuscript, additional characteristics and functions of meta-therapy were illuminated. We will summarize them briefly here with the expectation that they will be explored in the future. First, we propose that meta-therapy might serve a purpose akin to vocal stimulability testing, ${ }^{48,49}$ but from a more psychological or representational perspective. We have spent much time discussing meta-therapy and its ability to facilitate the patients' understanding of themselves, the process of vocal change, and so on. However, meta-therapy might also serve to facilitate the clinician's understanding of what voice-related mental representations need modifying, as well as which informational ingredients have the best potential for success with an individual patient. When meta-therapy is applied with a patient, the patient sends back information that serves as feedback about whether the clinician is providing information in the right manner, in the right place, in the right amount, at the right time. Information sent back from the patient can be obvious or subtle, fleeting or lasting, seemingly random or patterned. This complex material is precisely the type of information that human brains are wired to calculate: eye contact, nervous laughter, body posture, ease of movement, voice quality, speech rate, and so on. In short, how a patient responds to metatherapy is essentially representational (to apply an RTSS term) stimulability testing that can guide the clinician to the next thing they need to say or do. In voice therapy, clinicians talk colloquially about "the hook" as the way to motivate a person to "buy in" to voice therapy. This feedback from the patient to the clinician's meta-therapeutic dialogue is actively shaping the clinician's understanding of what "the hook" needs to be for that person in the context of the moment and their life. And, building on that notion, when patient feedback emerges from meta-therapy as a diagnostic probe, it serves as adjunctive diagnostic information so that the clinician can root out potential origins of their vocal impairments. Even if the vocal impairments are purely biomechanical in nature, the barriers to resolving them might yet be grounded in any number of representational mental states, perhaps stemming from negative affective states, conditioned stress responses, trauma, and so on. Thus, we propose that one broad aspect of meta-therapy is to essentially serve as "representational stimulability" mechanisms within a session, guiding clinicians to say and do the next best thing for the patient's long- and short-term success.

Another consideration, which we wish to propose briefly yet reserve for future elaboration, pertains to differences in outcomes between clinicians ostensibly delivering the same therapeutic approach. Some might refer to this as the "therapist effect" or simply reduce it to stylistic differences. For example, the timing of meta-therapy ingredients by the clinician is likely to shape the way the patient receives it, much akin to the timing of a joke, the delivery of criticism, or a request for a favor. The notion that subtle stylistic differences such as timing accounts for some variance in outcomes when comparing "super-shrinks" versus "pseudoshrinks" has been previously established. ${ }^{50,51}$ Also, multiple fields of treatment that rely upon behavior change have widely accepted that the patient's perception of the clinician's warmth, empathy, and trust is a key driver of patient outcomes. For example, high degrees of clinician empathy may help establish the trust required for a patient to share information about their voice disorder and related personal issues. Psychology researchers studying the "therapist effect" have examined empathy and warmth as ingredients, and seek to measure the "therapeutic alliance." ${ }^{52-55}$ These different features that a clinician might bring to the table could reasonably be viewed as stylistic in nature, thus begging the question of where one draws a line between style and skill. 
On the surface, it may appear that the success of meta-therapy might hinge on some features that are not readily encoded in the RTSS or taught programmatically. However, it is also possible and perhaps more likely the case that the features of meta-therapy are not yet specific enough in theory to fit the rules set forth by the RTSS. There is good reason for this lack of specificity. The clinical dialogue between clinician and patient is unquestionably complex, challenging to articulate, and simultaneously influenced by countless factors that are known, partially known, and fully unknown to one or both individuals. Further, meta-therapy likely unfolds differently as a function of numerous clinician and patient characteristics. For instance, gender identity, age, culture, and myriad other features of the individuals involved will come to bear on the clinical dialogue, perhaps even shifting from day to day as a function of environmental changes and current events. In theory, each of the above considerations could be systematically explored by applying a linguistic and supralinguistic analysis of the clinical dialogue that experienced versus novice clinicians share with their patients. Ideally, such a study would also explore outcomes between both groups to confirm whether, how, and to what extent experienced clinicians are in fact more effective and/or efficient in helping their patients achieve their goals. These and other questions about metatherapy should be reserved for future efforts in which the dialogue and delivery manner of metatherapy by expert clinicians is systematically investigated.

\section{CONCLUSION}

This paper aims to further characterize and define meta-therapy, a newly described and distinct sub-element of voice therapy that can be mapped onto the Rehabilitation Treatment Specification System (RTSS). Meta-therapy maps onto the RTSS framework at multiple levels, yet requires further characterization and investigation before it can be clearly operationalized. Inasmuch as meta-therapy is a clinical dialogue that is centered on a treatment theory and involves modifying a patient's cognitions, knowledge, beliefs, attitudes, intentions, and/or awareness, it seems clear that meta-therapy is a clinical entity that pervades speech-language pathology and other fields of allied health. Thus, future investigations should systematically parse and operationalize the various elements of meta-therapy, using the robust structure of the RTSS to guide the process.

\section{DISCLOSURES}

- Helou is a salaried employee of the University of Pittsburgh School of Health and Rehabilitation Sciences. She receives honoraria and royalties for speaking engagements on the topic of voice therapy and meta-therapy. She has a contractual engagement with and receives royalties from MedBridge, Inc.

- Gartner-Schmidt is a salaried employee of the University of Pittsburgh School of Medicine. She receives royalties from MedBridge, Inc., is a consultant on R01 DC01590601A1 for Optimization and Therapeutic Translation of Semi-Occluded Vocal Tract Techniques, and is adjunct professor, receiving annual honoraria, St. Mary's College, Notre Dame, IN.

- Hapner is a salaried employee of the University of Alabama, Birmingham. She receives honoraria and royalties for speaking engagements on the topic of voice therapy and metatherapy. She has a contractual engagement with MedBridge, Inc. as a consultant for the voice curriculum and for her work as an instructor on several topics on dysphonia that include discussions of meta-therapy. She is the co-author of the book, Voice Therapy Clinical Case Studies with Plural Publishing with several references to meta therapy within its case studies.

- Schneider is a salaried employee of the University of California San Francisco. She receives honoraria for planning of continuing education course and speaking engagements on the topic of voice evaluation and therapy. She receives royalties from MedBridge, Inc. for her work on education courses including Auditory Perceptual Voice Evaluation, Resonant Voice Tips and Tricks, and two courses on Singing Voice Evaluation and Treatment. She is the Professional Development Manager for the American Speech-Language and Hearing Association 
Special Interest Group 3: Voice and Upper Airway Disorders.

- Van Stan's work was supported by the National Institutes of Health (NIH) National Institute on Deafness and Other Communication Disorders and the Office of the Director's Office of Behavioral and Social Sciences Research via grant R21 DC016124 (PI: Dr. Jarrad Van Stan). The article's contents are solely the responsibility of the authors and do not necessarily represent the official views of the NIH. The funders had no role in the preparation, review, or approval of the manuscript, or the decision to submit the manuscript for publication. Jarrad Van Stan is also one of 9 copyright holders for the Manual for Rehabilitation Treatment Specification.

\section{CONFLICT OF INTEREST}

None declared

\section{REFERENCES}

1. Rangarathnam B, Gilroy H, McCullough G. Do patients treated for voice therapy with telepractice show similar changes in voice outcome measures as patients treated face to face? Evid Based Pract Briefs 2016;11.

2. Meerschman I, Van Lierde K, Claeys S, D'haeseleer E. The Patient's Opinion Regarding Different Service Delivery Models for Voice Therapy. Am J Speech Lang Pathol 2020;29(03):1466-1474. Doi: 10.1044/2020_AJSLP-19-00128

3. Meerschman I, Claeys S, Bettens K, Bruneel L, D'haeseleer E, Van Lierde K. Massed Versus Spaced Practice in Vocology: Effect of a ShortTerm Intensive Voice Therapy Versus a LongTerm Traditional Voice Therapy. J Speech Lang Hear Res 2019;62(03):611-630. Doi: 10.1044/ 2018_JSLHR-S-18-0013

4. Abrahamsson M, Millgård M, Havstam C, Tuomi L. Effects of Voice Therapy: A Comparison Between Individual and Group Therapy. J Voice 2018; 32(04):437-442. Doi: 10.1016/j.jvoice.2017.06.008

5. Desjardins M, Halstead L, Cooke M, Bonilha HS. A Systematic Review of Voice Therapy: What "Effectiveness” Really Implies. J Voice 2017;31(03):392. e13-392.e32. Doi: 10.1016/j.jvoice.2016.10.002

6. De Bodt M, Patteeuw T, Versele A. Temporal Variables in Voice Therapy. J Voice 2015;29(05): 611-617. Doi: 10.1016/j.jvoice.2014.12.001

7. Casper JK, Murry T. Voice therapy methods in dysphonia. Otolaryngol Clin North Am 2000;33
(05):983-1002. Doi: 10.1016/S0030-6665(05) 70259-0

8. Sellars C, Carding PN, Deary IJ, MacKenzie K, Wilson JA. Characterization of effective primary voice therapy for dysphonia. J Laryngol Otol 2002; 116(12):1014-1018

9. Rodríguez-Parra MJ, Adrián JA, Casado JC. Comparing voice-therapy and vocal-hygiene treatments in dysphonia using a limited multidimensional evaluation protocol. J Commun Disord 2011;44 (06):615-630. Doi: 10.1016/j.jcomdis.2011.07.003

10. Niebudek-Bogusz E, Sznurowska-Przygocka B, Fiszer Met al.. The effectiveness of voice therapy for teachers with dysphonia. Folia Phoniatr Logop 2008;60(03):134-141. Doi: 10.1159/000120290

11. Verdolini-Marston K, Burke MK, Lessac A, Glaze L, Caldwell E. Preliminary study of two methods of treatment for laryngeal nodules. J Voice 1995;9 (01):74-85

12. Ziegler A, Dastolfo C, Hersan R, Rosen CA, Gartner-Schmidt J. Perceptions of voice therapy from patients diagnosed with primary muscle tension dysphonia and benign mid-membranous vocal fold lesions. J Voice 2014;28(06):742-752. Doi: 10.1016/j.jvoice.2014.02.007

13. Watts CR, Hamilton A, Toles L, Childs L, Mau T. Intervention Outcomes of Two Treatments for Muscle Tension Dysphonia: A Randomized Controlled Trial. J Speech Lang Hear Res 2019;62(02): 272-282. Doi: 10.1044/2018_JSLHR-S-18-0118

14. Kapsner-Smith MR, Hunter EJ, Kirkham K, Cox K, Titze IR. A Randomized Controlled Trial of Two Semi-Occluded Vocal Tract Voice Therapy Protocols. J Speech Lang Hear Res 2015;58(03): 535-549

15. Helou L. Crafting the dialogue: Meta-therapy in transgender voice and communication training. Perspect ASHA Spec Interest Groups SIG 10 2017;2(02).

16. Speech Uncensored Podcast. "Meta-Therapy”: How Conversation Shapes Therapeutic Response with Leah B. Helou, PhD, CCC-SLPhttps://www.speechuncensored.com/podcastepisodes/s2e10-metatherapy-how-conversation-shapes-therapeutic-response-with-leah-b-helou-phd-ccc-slp

17. Helou L. Beyond Successful Voice Therapy Techniques and Tasks: The Concept of Meta-Therapy. In: Stemple JC, Hapner ER eds. Voice Therapy: Clinical Case Studies. Fifth.Plural Publishing, Inc.; 2019:28-31

18. Helou L. Meta-therapy: What's your framework? Presented at the: The Sin City Laryngology PreConference; February 2020; Las Vegas, NV

19. Helou L, Block C, Hirsch S. Transgender Voice and Communication Training. Presented at the: Transgender Voice and Communication Training (Independent educational workshop); September 19, 2015; Chicago, IL 
20. Helou L. Voice Training for the Transgender Client. Presented at: The Voice Therapy Conference; 2015 Pittsburgh, PA

21. Van Stan JH, Dijkers MP, Whyte Jet al. The Rehabilitation Treatment Specification System: Implications for Improvements in Research Design, Reporting, Replication, and Synthesis. Arch Phys Med Rehabil 2019;100(01):146-155. Doi: 10.1016/j.apmr.2018.09.112

22. Whyte J, Dijkers MP, Van Stan JH, Hart T. Specifying What We Study and Implement in Rehabilitation: Comments on the Reporting of Clinical Research. Arch Phys Med Rehabil 2018;99(07): 1433-1435. Doi: 10.1016/j.apmr.2018.03.008

23. Zanca JM, Turkstra LS, Chen Cet al.. Advancing Rehabilitation Practice Through Improved Specification of Interventions. Arch Phys Med Rehabil 2019; 100(01):164-171. Doi: 10.1016/j.apmr.2018.09.110

24. Hoffmann TC, Glasziou PP, Boutron Iet al.. Better reporting of interventions: template for intervention description and replication (TIDieR) checklist and guide. BMJ 2014;348:g1687. Doi: 10.1136/bmj.g1687

25. Whyte J, Dijkers M, Fasoli SEet al.. Recommendations for Reporting on Rehabilitation Interventions. Am J Phys Med Rehabil 2020;Publish Ahead of Print. Doi: 10.1097/PHM.0000000000001581

26. Van Stan JH, Whyte J, DuffyJRet al.. Rehabilitation Treatment Specification System: Methodology to identify and describe unique targets and ingredients. Arch Phys Med Rehabil 2020:S0003-9993(20) 31020-0. Doi: 10.1016/j.apmr.2020.09.383

27. Whyte J, Hart T. It's more than a black box; it's a Russian doll: defining rehabilitation treatments. Am J Phys Med Rehabil 2003;82(08):639-652. Doi: 10.1097/01.PHM.0000078200.61840.2D

28. Whyte J, Dijkers MP, Hart Tet al.. The Importance of Voluntary Behavior in Rehabilitation Treatment and Outcomes. Arch Phys Med Rehabil 2019;100 (01):156-163. Doi: 10.1016/j.apmr.2018.09.111

29. Hart T, Dijkers MP, Whyte Jet al.. A TheoryDriven System for the Specification of Rehabilitation Treatments. Arch Phys Med Rehabil 2019;100 (01):172-180. Doi: 10.1016/j.apmr.2018.09.109

30. Definition of meta $\mid$ Dictionary.com. www.dictionary.com. Accessed September 4, 2020. https:// www.dictionary.com/browse/meta-

31. Hume D, Millican PF. An Enquiry Concerning Human Understanding. Oxford University Press; 2007

32. Guarino N. The Ontological Level: Revisiting 30 Years of Knowledge Representation. In: Borgida AT, Chaudhri VK, Giorgini P, Yu ESeds.. Conceptual Modeling: Foundations and Applications: Essays in Honor of John Mylopoulos. Lecture Notes in Computer ScienceSpringer; 2009:52-67. Doi: 10.1007/978-3-642-02463-4_4
33. Andrade PA, Wood G, Ratcliffe P, Epstein R, Pijper A, Svec JG. Electroglottographic study of seven semioccluded exercises: LaxVox, straw, lip-trill, tonguetrill, humming, hand-over-mouth, and tongue-trill combined with hand-over-mouth. J Voice 2014;28 (05):589-595. Doi: 10.1016/j.jvoice.2013.11.004

34. Titze IR. Voice training and therapy with a semioccluded vocal tract: rationale and scientific underpinnings. J Speech Lang Hear Res 2006;49(02): 448-459. Doi: 10.1044/1092-4388(2006/035)

35. Roy N, Weinrich B, Gray SDet al.. Voice amplification versus vocal hygiene instruction for teachers with voice disorders: a treatment outcomes study. J Speech Lang Hear Res 2002;45(04):625-638. Doi: 10.1044/1092-4388(2002/050)

36. van Leer E, Connor NP. Patient perceptions of voice therapy adherence. J Voice 2010;24(04): 458-469. Doi: 10.1016/j.jvoice.2008.12.009

37. Halpern DF, Hansen C, Riefer D. Analogies as an aid to understanding and memory. J Educ Psychol 1990; 82(02):298-305. Doi: 10.1037/0022-0663.82.2.298

38. van Mersbergen M. Re-Acquainting yourself with Voice Therapy Techniques. Presented at the: Illinois Speech Language Hearing Association Convention; February 2015; Rosemont, IL

39. Iwarsson J. Facilitating behavioral learning and habit change in voice therapy-theoretic premises and practical strategies. Logoped Phoniatr Vocol 2015;40(04): 179-186. Doi: 10.3109/14015439.2014.936498

40. Behrman A. Facilitating behavioral change in voice therapy: the relevance of motivational interviewing. Am J Speech Lang Pathol 2006;15(03):215-225. Doi: 10.1044/1058-0360(2006/020)

41. van Mersbergen M, Lyons P, Riegler D. Vocal Responses in Heighted States of Arousal. J Voice 2017;31(01):127.e13-127.e19. Doi: 10.1016/j. jvoice.2015.12.011

42. Crow KM, van Mersbergen M, Payne AE. Vocal Congruence: The Voice and the Self Measured by Interoceptive Awareness. J Voice 2019:S0892-1997 (19)30293-0. Doi: 10.1016/j.jvoice.2019.08.027

43. Helou LB, Rosen CA, Wang W, Verdolini Abbott K. Intrinsic Laryngeal Muscle Response to a Public Speech Preparation Stressor. J Speech Lang Hear Res 2018;61(07):1525-1543. Doi: 10.1044/ 2018_JSLHR-S-17-0153

44. Dietrich M, Verdolini Abbott K. Vocal function in introverts and extraverts during a psychological stress reactivity protocol. J Speech Lang Hear Res 2012;55(03):973-987. Doi: 10.1044/10924388(2011/10-0344)

45. Dietrich M, Verdolini Abbott K, Gartner-Schmidt J, Rosen CA. The frequency of perceived stress, anxiety, and depression in patients with common pathologies affecting voice. J Voice 2008;22(04):472-488

46. Dietrich M, Andreatta RD, Jiang Y, Joshi A, Stemple JC. Preliminary findings on the relation 
between the personality trait of stress reaction and the central neural control of human vocalization. Int J Speech Lang Pathol 2012;14(04):377-389. Doi: 10.3109/17549507.2012.688865

47. Gillespie AI, Abbott KV. The influence of clinical terminology on self-efficacy for voice. Logoped Phoniatr Vocol 2011;36(03):91-99. Doi: 10.3109/14015439.2010.539259

48. Gillespie AI, Gartner-Schmidt J. Immediate Effect of Stimulability Assessment on Acoustic, Aerodynamic, and Patient-Perceptual Measures of Voice. J Voice 2016;30(04):507.e9-507.e14. Doi: 10.1016/ j.jvoice.2015.06.004

49. Bonilha HS, Dawson AE. Creating a mastery experience during the voice evaluation. J Voice 2012;26(05): 665.e1-665.e7. Doi: 10.1016/j.jvoice.2011.09.004

50. Miller S, Hubble MA, Duncan BL. The Secrets of Supershrinks: Pathways to Clinical Excellence psychotherapynetworker. Published 2013. Accessed September 4, 2020. /paper/The-Secrets-of-Supershrinks-\%3A-Pathways-to-Clinical-Miller-Hubble/ ef6dece61af4b9bb1ea3e0d26c255e8f17f612d9
51. Okiishi J, Lambert MJ, Nielsen SL, Ogles BM. Waiting for supershrink: An empirical analysis of therapist effects. Clin Psychol Psychother 2003;10 (06):361-373. Doi: 10.1002/cpp.383

52. Del Re AC, Flückiger C, Horvath AO, Symonds D, Wampold BE. Therapist effects in the therapeutic alliance-outcome relationship: a restricted-maximum likelihood meta-analysis. Clin Psychol Rev 2012;32 (07):642-649. Doi: 10.1016/j.cpr. 2012.07.002

53. Falkenström F, Granström F, Holmqvist R. Therapeutic alliance predicts symptomatic improvement session by session. J Couns Psychol 2013;60(03): 317-328. Doi: 10.1037/a0032258

54. Martin DJ, Garske JP, Davis MK. Relation of the therapeutic alliance with outcome and other variables: a meta-analytic review. J Consult Clin Psychol 2000;68(03):438-450. Doi: 10.1037/0022-006X. 68.3 .438

55. The conceptualization and measurement of therapeutic alliance: An empirical review - ScienceDirect. Accessed September 4, 2020. https://www.sciencedirect.com/science/article/pii/S02727 35808000858 